II.

\title{
Die ägyptischen Urkunden und das Eherecht der römischen Soldaten.
}

\author{
Von \\ Herrn Dr. Paul Meyer \\ in Berlin.
}

\section{A. Zum Eherecht der römischen Bürgersoldaten in Aegypten unter Trajan.}

1. Urkunde 140 des Berliner Museums.

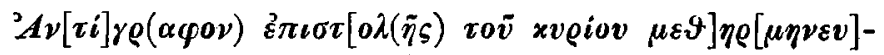
$\mu \varepsilon \dot{v \eta} \varsigma[\ldots \ldots \ldots . .$.

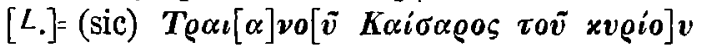

$\left.[\ldots] \pi \lambda i o v \quad A[\ldots \ldots \ldots \ldots \ldots] \sigma \tau \iota x o v \quad \lambda(?)^{1}\right)$

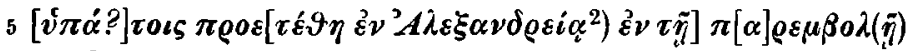

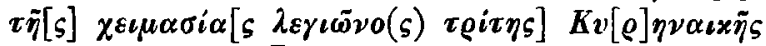

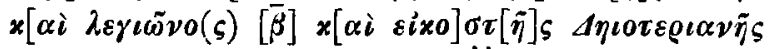

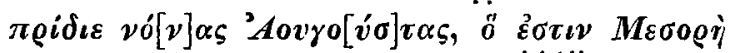

$\overline{\iota \alpha}$ हैे $\pi \varrho \iota \nu \times \varepsilon[\pi] i 0 \iota[s]$.

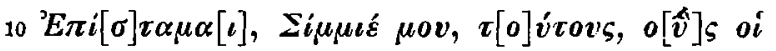

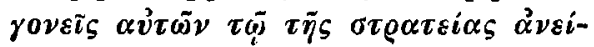

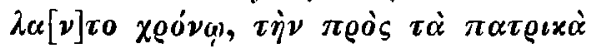

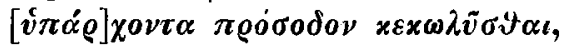

1) Die Consulnamen haben hier sicher gestanden, obwohl der Raum sehr klein ist. Eine Ergänzung der Namen ist (zumal mir nur die Photographie zur Verfügung steht) unmöglich. Ich lese v. 4 ungefähr ( $\iota$ od. $\eta$ ?) dıov ri- $\sigma \tau \iota x o v \lambda .-{ }^{2}$ ) cf. Edictum Claudii de civitate Anaunorum 2 sq.: idibus Martis Bais in praetorio edictum - proposi-

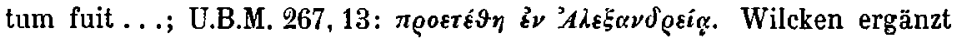

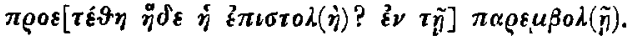




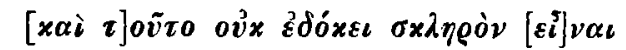

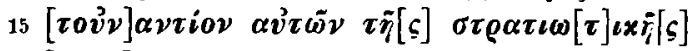

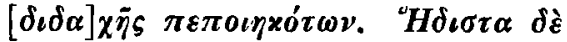

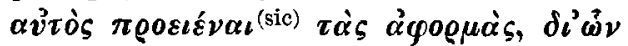

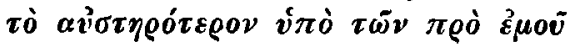

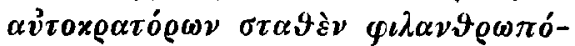

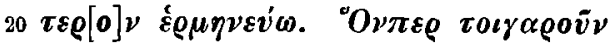

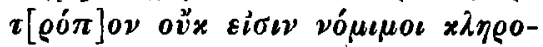

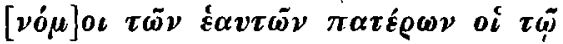

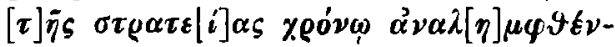

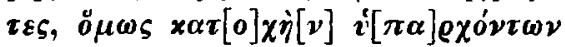

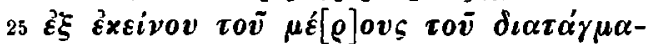

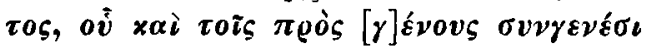

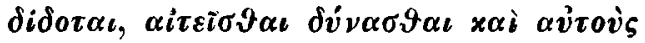

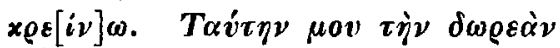

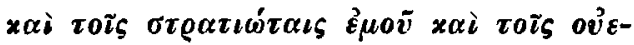

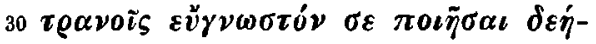

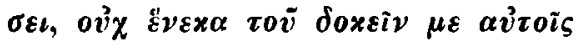

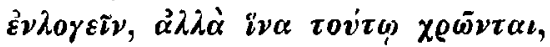
$\dot{\varepsilon} \dot{\alpha} \nu \dot{\alpha}^{\gamma} \gamma \nu \tilde{\omega} \sigma$.

\section{Uebersetzung :}

Abschrift der Uebersetzung einer kaiserlichen epistula .... Im [10.(?)] Jahre unseres Herrn und Kaisers Trajan unter dem Consulate des ? erfolgte die öffentliche Bekanntmachung in Alexandria in dem Winterlager der legio III. Cyrenaica und der legio XXII. Deiotariana am 4. August, nach ägyptischer Rechnung am 11. Mesore, im Hauptquartier.

Ich weiss, mein Simius, dass die Kinder, welche ibren Eltern während der Dienstzeit des Vaters geboren sind, des Intestaterbrechtes gegenüber dem Letzteren beraubt sind; und dies scheint mir nicht hart zu sein im Hinblick darauf, dass die Eltern den militärischen Vorschriften entgegengehandelt haben. Mit Freuden will ich aber die Gelegenbeit ergreifen, um die allzustrengen Bestimmungen meiner Vorgänger in milderer Weise auszulegen. Ich bestimme deshalb, dass, obschon die während der Dienstzeit ihrer Väter geborenen Kinder nicht legitimi derselben sind, ihnen dennoch erbrechtlich dieselbe Stellung gewährt werden soll, wie den in der 
Classe unde cognati zur bonorum possessio Berufenen. Dieses von mir ertheilte Privileg bitte ich dich sowohl meinen Soldaten als den Veteranen bekannt zu machen, nicht als ob ich es ibnen anrechnen wollte, sondern damit sie, falls sie es nicht wissen sollten, Gebrauch davon machen. -

Die Urkunde enthält eine in Briefform gefasste Dienstinstruction und Vollmacht des Kaisers Trajan an den praefectus Aegypti C. Sulpicius Simius, den obersten militärischen Befehlshaber und Verwaltungsbeamten des Landes. Das Mandat, das in das Jahr 106 oder $107 \mathrm{zu}$ setzen ist (am 4. August), bezieht sich auf die Kinder der activen Soldaten und Veteranen der legio III. Cyrenaica und legio XXII. Deiotariana ${ }^{1}$ ).

Wir haben es hier mit einer Abschrift aus den Originalacten zu thun, die zu bestimmtem Zweck angefertigt, übersetzt und in das Archiv des arsinoitischen Gaues aufgenommen ist. Das Mandat, an und für sich eine geheime Instruction des Kaisers an den Beamten, ist auf ausdrücklichen Befehl des Absenders (v. 28 sqq.) im Hauptquartier in Alexandria öffentlich bekannt gemacht (v. 5 sqq.) und steht dadurch auf gleicher Stufe mit den orationes principis in castris praetoriis recitatae (frgm. Vat. $\S 195$; Cod. Theod. 7, 20, 2). Eine beglaubigte Abschrift der Bekanntmachung ist in das Archiv des Präfecten aufgenommen; unsere Urkunde ist die griechische Uebersetzung dieses Actenstücks.

Das kaiserliche Mandat hat also folgende Veränderungen in Bezug auf die äussere Form und die einzelnen Instanzen durchgemacht:

1. Lateinische epistula des Kaisers an den praef. Aeg.

2. Oeffentliche Bekanntmachung im Hauptquartier zu Alexandria in der (lateinischen) Armeesprache.

3. Officielle Abschrift derselben für das Archiv des praef. Aeg.

4. Private griechische Uebersetzung.

3. wird ungefähr folgendermassen gelautet haben :

N. N. cos. pridie nonas Augustas Alexandriae in castris hibernis legionis III. Cyrenaicae et legionis XXII. Deiotarianae

1) Die nähere Begründung und Ausführung der historischen Seite habe ich in einem Aufsatze im Hermes (XXXII, 215 f.) gegeben. 
Die āgypt. Urkunden und das Eherecht der löm. Soldaten.

epistula imperatoris Caesaris Nervae Traiani Augusti proposita est in principiis:

Traianus Simio suo (salutem dicit). (Folgt der Inhalt.)

In der uns vorliegenden Uebersetzung (4.) ist hinzugefügt

a) die Bezeichnung als Abschrift und Uebersetzung (v. 1),

b) die ägyptische Datirung nach $\alpha$ ) Regierungsjahren des

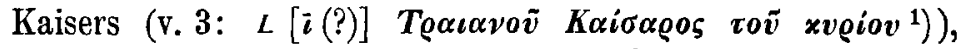

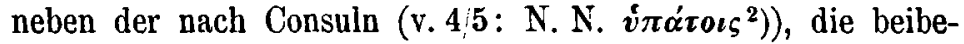
balten $\left.{ }^{3}\right)$, und $\beta$ ) ägyptischem Kalender, neben der zugleich bei-

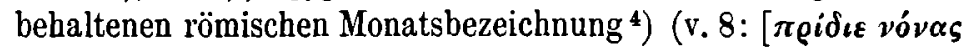

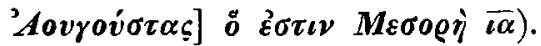

Ausgelassen ist dagegen die Anrede des Kaisers in der epistula an den praef. Aeg.

Gehen wir jetzt auf den Inhalt der indulgentia ein! Sie enthält eine erbrechtliche Vergünstigung für Kinder aus geschlechtlichen Verbindungen, die nicht erst während, sondern schon vor der Dienstzeit geschlossen sind (v. $10 \mathrm{sqq}$.).

Für eigentlicheSoldatenkinder gelten andere Bestimmungen, als für ihre vor oder $\mathrm{nach}$ der Dienstzeit geborenen Geschwister: im Gegensatz $\mathrm{zu}$ diesen, die legitim sind, entbehren sie als illegitim eines Intestaterbrechtes gegenüber ihrem Vater (v.12 sqq.), der sich in Widerspruch gesetzt hat mit den Militärgesetzen (v. $15 \mathrm{sq}$.). Wir haben es also mit einer Ausnahmestellung zu thun, welche die Militärgesetze (leges Iuliae et

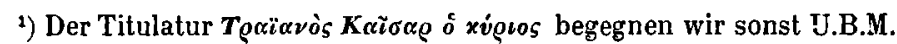
$68 ; 213 ; 50 ; 101$ in nicht amtlichen; 360 in einem amtlichen Schriftstück, ist aber nicht officiell. $-{ }^{2}$ ) Für die Uebersetzung des lateinischen abl. abs. durch den griechischen dat. abs. finden sich auch sonst Analogien: Kaibel, Inscr. 1055b; 2090; 1045; (830); U.B.M. 326 II; 586, 29; s. auch Monum. Ancyr. int. gr. 2 p. 198 (ed. Momms.). - 3) Auch U.B.M. 326 II

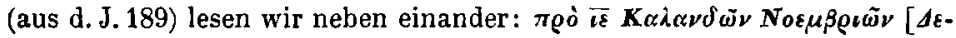

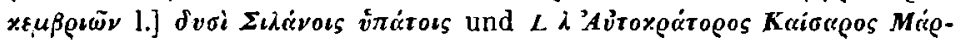
xov Aíphiov Kouódov 'Av7wveivov. Die doppelte Datirung ist auch hier zu erklären aus der griechischen Uebersetzung des lateinischen Originales unter Hinzufügung der ägyptischen Datirung. Es sind dies (U.B.M. $140 \mathrm{u}, 326)$ die beiden einzigen uns bekannten ägyptischen Urkunden vor dem 4. Jahrhundert, in denen sich Datirung nach Consuln findet. - ${ }^{4}$ Ebenso U.B.M. 113, v. 8/9. Dagegen U.B.M. 265 ist die römische Datirung direct durch die ägyptische ersetzt, U.B.M. 326 der ägyptische Monat nicht hinzugefügt. 
Papia Poppaea) den Soldaten zuweisen: das für die Allgemeinheit geltende ius commune ist während der Dienstzeit suspendirt, ein ius singulare tritt in Kraft.

Welche geschlechtlichen Verbindungen können nun an und für sich hier in Betracht kommen?

Sehen wir von der Frage der Rekrutirung der ägyptischen Legionen in trajanischer Zeit ab und erörtern alle Möglichkeiten, die hinsichtlich der Nationalität und dementsprechend der Art der geschlechtlichen Verbindung der Legionare vor ihrer Dienstzeit denkbar sind. Sie können vor ihrem Eintritt in die Legion 1. cives R., 2. peregrini gewesen sein. Die vorher geschlossene Verbindung kann daher sein

$1^{2}$. matrimonium iustum iuris civilis;

$1^{\text {b. }}$ matrimonium iniustum (s. Meyer, Concubinat $32 ; 58$ );

1. Concubinat;

2a. matrimonium iustum iuris peregrini;

$2^{\text {b }}$. matrimonium iniustum zwischen cives Romanae und peregrini ohne conubium (s. Concubinat 31): diese Verbindung kommt nicht in Betracht.

Erwägen wir zuerst $1^{b}$ : die aus solcher Verbindung stammenden Kinder stehen nicht in der potestas des Vaters, haben kein legitimum ius ihm gegenüber, in Folge dessen auch kein Intestaterbrecht.

2a. Mit dem Eintritt in die Legion wird der Mann civis R, die Frau bleibt peregrina, da das Militärgesetz eine Ehefrau nicht kennt. Aus der bisher vollgiltigen peregrinen Ehe ist also ein matrimonium iniustum geworden (s. $1^{\text {b }}$ ). Die vor der Dienstzeit geborenen Kinder hatten als peregrini ihrem pater peregrinus gegenüber volles Erbrecht, die dagegen während derselben zur Welt gekommenen haben als peregrini ihrem pater civis $R$. gegenüber kein Intestaterbrecht. Es besteht also zwar ein Unterschied zwischen den vor und während der Dienstzeit geborenen, aber deshalb können die aus einer solchen Verbindung stammenden Soldatenkinder hier nicht gemeint sein, weil sie erbrechtlich nicht anders gestellt sind als alle sonstigen aus solchem matrimonium iniustum stammenden Kinder.

$1^{\text {c}}$. Ebensowenig kommt ein etwa vor dem Diensteintritt eingegangener Concubinat in Betracht. Von einem Intestat- 
erbrecht der Concubinenkinder ist niemals bis auf Justinian (s. Concub. 154) die Rede; es braucht diese Thatsache also nicht besonders betont $\mathrm{zu}$ werden.

Demnach bleibt nur die Annahme eines vor dem Eintritt in die Legion ${ }^{1}$ ) schon bestehenden matrimonium iustum iuris civilis $\left.^{2}\right)\left(1^{2}\right)$ übrig, dessen rechtliche Wirkungen während der Dienstzeit suspendirt werden (Concub. 106 f.; 114). Die in dieser Zeit geborenen Kinder sind rechtlich den Concubinenkindern gleichgestellt, nicht legitimi heredes ihres ,natürlichen" Vaters, haben als extranei kein Intestaterbrecht: dem entspricht der Wortlaut von v. 20 sqq.

Diesen im suspendirten matrimonium iustum geborenen Kindern der activen und inactiven Soldaten der legio III. Cyrenaica und legio XXII. Deiotariana gewährt Trajan die bonorum possessio unde cognati (v. 24 sqq.; s. Mitteis, Hermes 1895, 614). Sie bleiben unehelich, werden nicht Agnaten des Vaters, heredes legitimi (v. 20 sqq.), aber ibre Stellung als extranei wird insofern modificirt (v. $16 \mathrm{sqq}$.), als sie zum „Vater" in ein Cognationsverbältniss treten ${ }^{3}$ ).

\section{Papyrus Cattaoui 4 ) Col. II.}

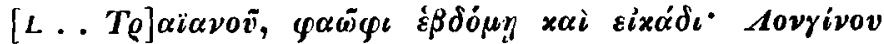

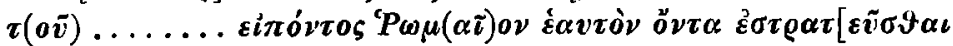

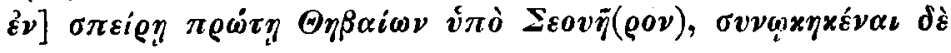

2) Ehe vor dem Diensteintritt s. Concubinat 102 ; ferner C.I.L. III, 6092. Verlobung vor dem Eintritt und Zusammenleben während der Dienstzeit (als sponsus und sponsa) im "legitimen Concubinat" C.I.L. VIII, 2857; 3065. - Wir finden Diensteintritt mit 23 Jahren C.I.L. VIII, 2103, 3148; mit 24 Jahren G. VIII, 3174, 3271; mit 25 Jahren C. VI, 2614; mit 32 Jahren C. VI, 2473; mit 34 Jahren C. III, 6596. - ${ }^{2}$ ) Dies bestätigt die uns aus anderen ägyptischen Urkunden bekannte Rekrutirung der ägyptischen Legionen in trajanischer Zeit: das Rekrutirungsmaterial derselben war schon am Anfang des 2. Jahrhunderts vor dem Eintritt in die Legion zum grössten Theile im Besitze des Bürgerrechts. Es setzte sich in erster Linie zusammen aus den ex castris und den Veteranen der auxilia und der Flotten (U.B.M. 113; 265), daneben wohl auch aus den vioi

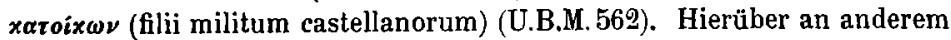
Orte! - ${ }^{3}$ ) Ueber die Bedeutung dieser Massregel in Hinsicht auf ägyptische Verhältnisse und die Regierungsmaximen des Trajan s. die Zusammenfassung S. ó 4 ff. $-{ }^{4}$ ) Dieser im Privatbesitz befindliche Papyrus ist von Botti in der Rivista Egiziana 1894 p. 529 sqq. herausgegeben -

Zeitschrift für Rechtsgeschichte. XVIII. Rom. Abth. 


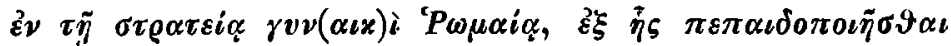

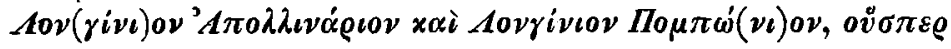

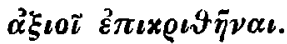

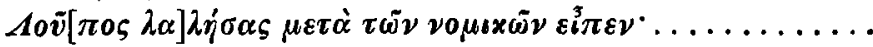

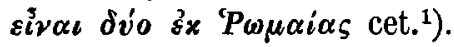

\section{Uebersetzung:}

Im . . . . Jahre des Trajan, am 27. Phaophi.

Longinus . . . . . giebt an, dass er, ein geborener civis R., seine Dienstzeit in der cohors I. Thebaeorum unter Severus absolvirt habe; während derselben habe er aber mit einer civis Romana zusammengewohnt, die ihm 2 Söhne, Longinius Apollinaris und Longinius Pomponius, geboren. Er beantragt nun, dieselben anzuerkennen.

Lupus entscheidet nach Berathung mit den rechtskundigen Beisitzern : .......

Wir haben einen Rechtsfall vor uns, der von dem praef. Aeg. M. Rutilius Lupus entschieden wird. Dieser fungirte, wie Hermes XXXII, $216 \mathrm{ff}$. dargelegt, mindestens vom Jahre 115 - beim Ausbruch des jüdischen Aufstandes war er schon im Amt - bis zum Ende der Regierung des Trajan. Unsere Urkunde fällt in oder kurz vor das Jahr 115 (aus diesem Jahre stammt die folgende Columne (III) des Papyrus).

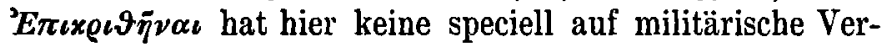
hältnisse bezügliche technische Bedeutung, wie ich sie Philologus LVI, $206 \mathrm{ff}$. auseinandergesetzt habe. Vielmehr wird das Wort in dem nicht übertragenen Sinne von probari, confirmari $=$ bestätigen, billigen, anerkennen (s. Fiebiger, de classium Italicarum historia et institutis [Leipziger Studien XV]

leider unvollständig - und von Scialoja im Bullettino dell'Istituto di diritto romano 1895 (VIII), 155 sqq. unverändert abgedruckt und durch eine auf antiquirtem Standpunkt stehende Abhandlung über das Eherecht der Soldaten vermehrt worden.

1) Botti speist uns, ohne den Wortlaut der Entscheidung weiter mitzutheilen, mit den Worten ab: „inutile il resto della sentenza, che è troppo lacunosa." Sein Resumé: „il magistrato non ammette la romanità della convivente od almeno que siano proprio di lei (lontana probabilmente dal marito che militava in Nubia) i due figli" lässt sich daher in Bezug auf seine Richtigkeit nicht controlliren. Sicher ist jedenfalls, dass die Kinder nicht als eheliche anerkannt werden. 
Die ägypt. Urkunden und das Eherecht der röm. Soldaten.

p. 422) gebraucht. Die Kinder sollen also ,als eheliche anerkannt werden".

Svvoıxeiv bedentet hier ebensowenig wie bei Dio und Herodian ,geschlechtlich beiwohnen" (s. Concub. 96 f., dagegen Kübler in dieser Zeitschr. XVII, 364), vielmehr ,zusammenwohnen" schlechthin, wie dies auch Scialoja 1.l. p.165 erklärt.

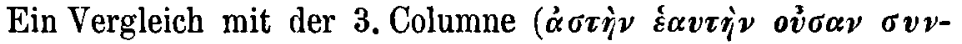

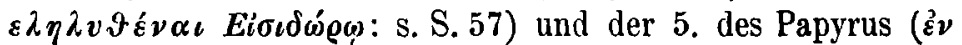
$\tau \tilde{\omega} \sigma v \nu \beta \iota \omega ́ \sigma \varepsilon \omega \varsigma \chi \varrho o ́ v \omega)$ zeigt uns, dass in unserer Urkunde der Nachdruck auf dem örtlichen Zusammenwohnen liegt. Wir finden also schon unter Trajan ausnahmsweise, was erst Severus den Soldaten allgemein gewährte.

Ueber die Bedeutung von vouıxós s. Mommsen in dieser Zeitschr. XII (1892), 293.

Longinus hat demnach während seiner Dienstzeit in der cohors I. Thebaeorum, die damals in Nubien stand, mit einer civis Romana „zusammengewohnt". Er beantragt die rechtliche Anerkennung seiner aus dieser Verbindung geborenen Söhne. Dieselbe ist also als "legitimer Concubinat" (s. Conc. $108 ; 110 ; 114)$ zu bezeichnen, der nach Beendigung der Dienstzeit in matrimonium iustum verwandelt ist. Von nachträglicher Legitimation der Kinder kann nicht die Rede sein: der Antrag ist also zweifellos von Lupus abgelehnt.

$$
\text { 3. U.B.M. } 114 \text { Col. I v. 5-13 }{ }^{1} \text { ). }
$$

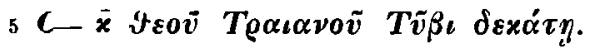

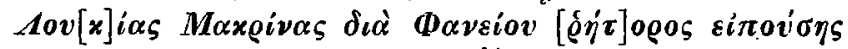

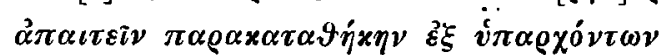

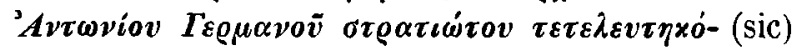

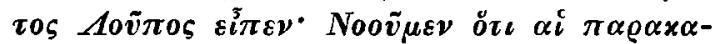

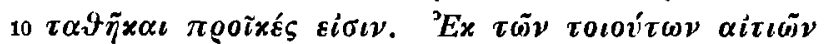

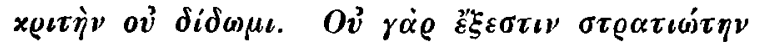

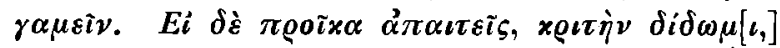

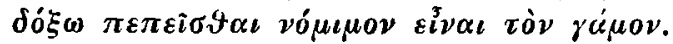

Uebersetzung:

Im 20. Jahre des divus Traianus am 10. Tybi. Auf die Forderung der durch den Rechtsbeistand Phaneus

1) Kurz behandelt von Mitteis (Hermes 30 [1895], 584f.). 
vertretenen Lucia Macrina, ihr aus der Hinterlassenschaft des Soldaten Antonius Germanus ein depositum zurückzuerstatten, entscheidet Lupus: Es handelt sich hier nicht um einen Deposital-, sondern um einen Dotal-Vertrag. Unter sothanen Umständen kann ich keine richterliche Entscheidung gewähren; denn der Soldat hat kein ius conubii. Stellst du aber die actio rei uxoriae an, dann will ich deinem Klageantrag nachgeben unter der Fiction, es handle sich um ein matrimonium iustum. -

In der U.B.M. 114 haben wir es mit den Abschriften mehrerer Entscheidungen zu thun, die sich auf die eherechtlichen Verbältnisse der Soldaten während der Dienstzeit beziehen. Sie sind wohl, um die Entscheidung in ähnlichen Fällen zu erleichtern, bei irgend einer Gelegenheit (nach dem Jahre 134, aus dem die dritte Entscheidung stammt: s. S. 63) zusammengestellt und zur jederzeitigen Benutzung dem Archiv von Arsinoe einverleibt.

Col. I v. 14 sqq. und Col. II (s. S. 61 ff.) haben wir es mit einem Auszug aus dem Journal (s. Wilcken, Philologus 1894, 107) eines vom praef. Aeg. Sex. Petronius Mamertinus delegirten

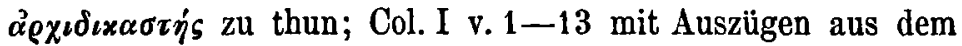
Journal des oben erwähnten praef. Aeg. M. Rutilius Lupus.

Die Entscheidung des hier in Betracht kommenden Falles (v. 5-13) ist vom 5. Januar 117 datirt. Sie betrifft die rechtlichen Beziehungen $z$ wischen einem verstorbenen Bürgersoldaten und einer civis Romana, die sich während der Dienstzeit des Ersteren ergeben haben. Der Statthalter geht von dem für das Militärrecht grundlegenden Satze aus: der Soldat hat kein ius conubii; macht dann aber von diesem in jener Zeit sonst niemals durchbrochenen Princip eine Ausnabme.

Die beiden obengenannten Personen haben bei Eingehung ihrer Verbindung einen Vertrag in Form eines depositum geschlossen und dadurch den dotalrechtlichen Charakter zu verdecken gesucht.

Wir haben es hier mit einer specifisch ägyptischen Sitte zu thun, die sich auch unter den ägyptischen Griechen einbürgerte. Der Gebrauch schriftlicher Ehecontracte ist obligatorisch. Oijx

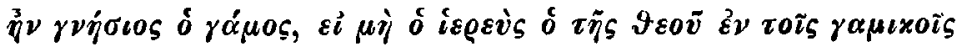

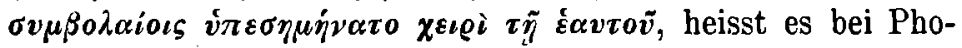




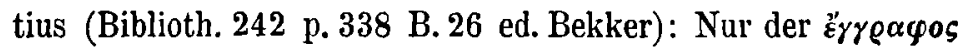
ráuos ist giltige Ehe (im Gegensatz zum römischen Recht) (s. Papyr. Rainer n. 1521-1524=Wessely, Corp. Pap. Rain. I n. 28). Einen regelmässigen Bestandtheil der $\gamma \alpha \mu \iota x \dot{\alpha} \sigma v \mu \beta \dot{\lambda} \lambda \alpha \iota \alpha$ bildet eine fictive Mitgift, welche der Mann empfangen soll, die aber in Wahrheit ein verschleiertes Brautgeschenk ist, das der Mann zu geben verspricht. Es ist gekleidet meistens in die Form eines Darlehns als allgemeinster Art des Credites (Beispiele s. Wessely, 1. 1. n. 21 sqq.; Pap. Cattaoui, Col. I und V; U.B.M. 114 I v. 14 sqq.: s. S. 61 ff.), aber auch in die eines Depositum (wie in unserem Fall und Wessely 1.1. n. 29). Solche Eheverträge repräsentiren also ein schriftliches Zahlungsversprechen, bei dem die causa obligationis nebensächlich ist; es sind abstracte Literalverträge, die $\mathrm{zu}$ vergleichen sind der römischen stipulatio (s. Mitteis, Reichsrecht u. Volksrecht $468 \mathrm{ff}$.).

Das ägyptisch-griechische Recht erkennt solche Verträge sowohl ehe- als vertragsrechtlich an und dementsprechend auch die römische Praxis, die gerade auf dem Gebiete des Eherechtes die ägyptischen Eigenthümlichkeiten möglichst schonte (Mitteis I. 1. 57).

Anders verhält es sich aber, sobald Soldaten in Betracht kommen, deren eheliche Verbindungen von den Militärgesetzen ignorirt werden.

Nicht nur peregrine (wie wir später sehen werden), sondern auch Bürgersoldaten scheinen sich mit Vorliebe solcher fingirter vermögensrechtlichen Verträge bedient zu haben, um dem strengen Militärgesetz ein Schnippchen zu schlagen. Gestützt auf die ägyptisch-griechische Sitte hofften sie wenigstens auf Anerkennung des Vertrages, d.h. auf Gewährung der aus dem Ehevertrage für die Frau und die Erben des Mannes (s. Mitteis, Hermes l. 1.) entspringenden vermögensrechtlichen Vortheile.

Ein wie gewöhnliches und übliches Requisit der römischen Soldaten solche Verträge geworden waren, zeigen uns die Worte des Papyr. Cattaoui Col. V (vom 23. November 136):

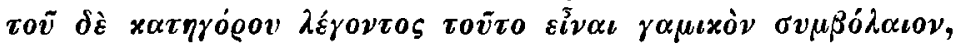

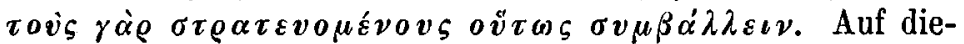
sen Einwand des Klägers entscheidet dort der Richter: $\tau \grave{o} .$.

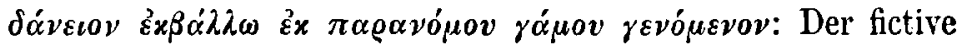


Vermögensvertrag ist gegrïndet auf die geschlechtliche Verbindung des Soldaten; Ehe eines solchen existirt vor dem Militärgesetz nicht. Deshalb ist weder ein Dotal- noch ein Darlehnsvertrag zu Stande gekommen; der abstracte Literalvertrag (welches auch die causa obligationis sein möge) ist nichtig.

Dementsprechend müsste Lupus auch in unserem Fall von Rechtswegen urtheilen und sowohl die actio depositi als die actio rei uxoriae abweisen, wie er denn auch erklärt: $\varepsilon^{3} x \tau \omega \nu$

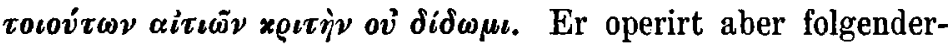
massen: Ein Vermögensvertrag liegt nicht vor, die Contractsklage ist daher abzuweisen ${ }^{1}$ ). Gemäss den Militärgesetzen liegt auch kein Dotalvertrag vor; denn aus einer von einem Soldaten geschlossenen geschlechtlichen Verbindung ergeben sich keine rechtlichen Wirkungen. Giebt aber die Klägerin durch Anstellung der actio rei uxoriae zu, dass es sich bei der Forderung um eine (fictive) dos, nicht um ein depositum handelt, dann will ich einmal Gnade vor Recht walten lassen und in diesem einen Fall vom Eheverbot für die Soldaten absehen.

Damit soll kein Präcedenzfall geschaffen werden für die während der Dienstzeit der Soldaten bestehenden Verhältnisse. Immerhin aber bleibt diese Entscheidung charakteristisch für die unter Trajan den Soldaten und speciell der ägyptischen Landessitte gegenüber an den Tag gelegte Connivenz.

Gerade in Aegypten bestand, wie in keinem anderen Lande, ein inniges Band zwischen den Landesbewohnern und den Soldaten. Sie waren eng verwachsen mit ägyptischen Sitten und Gebräuchen.

Die Soldateska der Ptolemaeer hatte im Anschluss an

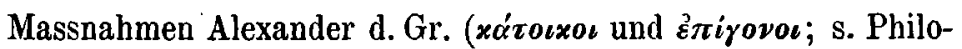
logus LVI, $193 \mathrm{ff}$.) in Bezug auf ihren Verkehr mit dem weiblichen Geschlecht, ein sich daraus ergebendes Familienleben und rechtliche Wirkungen immer grosse Freiheiten genossen.

1) In dem von Mitteis, Hermes I. l., angeführten Fall D. 16, 3, 27 liegt umgekehrt ein giltiger Depositalvertrag vor. Dementsprechend wird die actio depositi de peculio gegen den Eigenthümer des Sclaven gewährt, während bei contubernium (wie es dort zwischen ingenua und servus vorliegt) natürlich von einer Dotalklage nicht die Rede sein kann. 
Wohl oder übel mussten nach der Occupation des Landes die Römer diesen Gepflogenheiten (s. de bello civ. 3, 110; Tacit. hist. 3, 47) Rechnung tragen.

So bildet Aegypten den Ausgangspunkt für Massnahmen der römischen Kaiser auf militärischem Gebiete, die erst allmählich auf das übrige Reich übertragen wurden. Es waren ptolemäische Einrichtungen, die sie in ein neues Gewand kleideten: Unter Tiberius wurde hier das Institut der ex castris ins Leben gerufen; unter Augustus finden wir, ebenso wie unter den Ptolemaeern, Leute ausländischer Nationalität, die

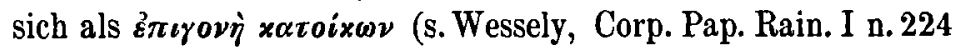
v. 3) bezeichnen. Im Laufe des 1. Jahrhunderts der Kaiserzeit tritt an ihre Stelle die in mancher Hinsicht privilegirte Classe der vioi xacoixwy, deren Listen aus trajanischer Zeit uns U.B.M. 562 v. 14 sqq. begegnen. Im 3. Jahrhundert treffen wir einen erblichen Soldatengrundbesitzerstand an allen Grenzen des Reiches als milites castellani (Militärdiplom n.90; s. Concub. $120 \mathrm{ff}$.).

Es ist ein gerade den Massnahmen Trajans zu Grunde liegendes Bestreben, jedem Lande seine Eigenart möglichst ungeschmälert zu lassen. Dies Bestreben äussert sich in Bestimmungen und Entscheidungen, wie sie uns in dem Mandat an Simius, in den beiden aus der Präfectur des Lupus stammenden Urkunden vorliegen. Sie sind specieller und beschränkter Natur und haben, wie so Manches aus der Regierungszeit des Trajan, keinen festen Bestand gehabt.

Der Kaiser gewährt den im suspendirten matrimonium iustum geborenen Kindern der Soldaten der legio III. Cyrenaica und legio XXII. Deiotariana, die jetzt zum grössten Theil aus Aegyptern rekrutirt wurden, die bonorum possessio unde cognati ${ }^{1}$ ); der unter ihm amtirende praef. Aeg. Lupus giebt

1) Mit dieser Verfügung stehen in gar keinem Zusammenhang die Vergūnstigungen, die nach Plinius panegyricus c. 37 sqq. Trajan, die Bestimmungen Nervas erweiternd, den novi cives gewãhrt. (Mitteis, Hermes 1. l. S. 614 glaubt einen Zusammenhang zu erkennen.) Plinius spricht von Massregeln des Kaisers, durch welche er allen Eltern resp. Kindern, die per Latium oder durch kaisertiches Rescript das Bürgerrecht erhalten hatten, gegenseitige Befreiung von der vicesima hereditatium et legatorum auch ohne Ertheilung der iura cognationum und ohne redactio in patriam potestatem (die nicht etwa fictiv aufzufassen 
der "Frau" eines verstorbenen Soldaten die actio rei uxoriae; wir finden zu gleicher Zeit einen Soldaten der cohors I. Thebaeorum, der während der Dienstzeit mit der "Frau“ zusammengewohnt hat.

Das Mandat Trajans enthält eine nur den beiden in der Vorstadt von Alexandria, in Nicopolis, kasernirten ägyptischen Legionen ertheilte Vergünstigung: den beiden einzigen Truppentheilen neben den cohortes praetoriae und urbanae in Rom und Lyon ${ }^{1}$ ), die eine städtische Garnison hatten. Wie diese in eherechtlicher Beziehung schon im 1. Jahrhundert vor den Legionen bevorzugt waren (s. Conc. \$ 30), so erbalten durch Trajan die ,ägyptischen Gardetruppen" analoge Privilegien.

Ob nach Schaffung der legio II. Traiana Fortis im Jahre 108 die Soldaten derselben die gleichen Vergünstigungen erhielten, muss dahingestellt bleiben. Von einer Erneuerung der Bestimmung durch die folgenden Kaiser, wie dies hinsichtlich

ist) zu Ungunsten des Fiscus gewährt. Es handelt sich 1. um testamentarisches Erbrecht (Dio 55, 25), 2. um Neubürger, deren ursprūnglich peregrines matrimonium nach der Bürgerrechtsertheilung in matrimonium iustum iuris civilis verwandelt worden war. - Ich muss Huschke (Gaius, Leipzig 1855 S. 20 ; s. auch Voigt, ius naturale III, 1157 n.1793; eine entgegengeseizte Ansicht vertreten Francke, Geschichte Trajans S. 446 ff.; de la Berge, Essai sur le règne de Tr. p. 141) darin beipflichten, dass „Plinius nur römische Bürger als Eltern oder Kinder vor Augen hat, welche von römischen Bürgern als Eltern oder Kindern erbten". (Dafür spricht auch c. 37: etiamsi cognationum iura non recepissent, quum civitatem adipiscerentur.) $\mathrm{Ob}$ er aber auch Recht hat mit der allgemeinen Behauptung, dass nur von Römern an Rōmer vererbte Vermögen der Steuer unterliegen, will ich dahingestellt sein lassen. Es ist richtig, dass der „Zwanzigste" nur von römischen Bürgern entrichtet wird (s. Bachofen, Ausgewählte Lehren des röm. Civilrechts, Bonn 1848 S. 333). Peregrine haben Bürgern gegenüber auch kein testamentarisches Erbrecht (Gaius II, 110; 275); eine Ausnahme gestattet nur Antoninus Pius für liberi peregrini gegenüber dem pater civis $\mathbf{R}$. (Pausan. 8, 43, 5). Erbschaften peregriner Rechtsgenossen gegenüber geniessen Peregrine Immunität; eine Ausnahme besteht nur für die cives Alexandrini (Pap. Cattaoui Col. III : s. S. 60). Caracalla will mit seiner Bürgerrechtsertheilung vor Allem die Peregrinen der Steuer unterwerfen. Letztwillige Zuwendungen von Peregrinen an Römer aber haben wohl schon vorher keine Steuerfreiheit genossen (vgl. z. B. den Cic. ad famil. 13, 30, 1 angeführten Fall aus republicanischer Zeit).

1) Dort lag die coh. XIII. urbana (Tacit. Ann. 3, 41, hist. 1, 64; Annal. dell'Ist. 1853, 74; Kieler Monatsschrift 1853, 651). 
der libera testamentifactio der Soldaten durch Titus, Domitian, Nerva, Trajan geschah, die damit ein fester Bestandtheil der Dienstordnung wurde, hören wir nichts. Wir haben es wohl mit einer noch unter Trajan wieder aufgehobenen indulgentia zu thun, die in allgemeinem Umfang wieder aufgenommen wurde am Ende des 2. Jahrhunderts durch die Uebertragung des Institutes der ex castris auf das suspendirte matrimonium iustum iuris civilis (Conc. 111).

In noch höherem Grade gilt dieser ephemere Charakter von den Thatsachen, die wir aus den anderen beiden Urkunden kennen lernen. Jedenfalls aber sind sie bezeichnend für die Regierung Trajans und die Sonderstellung der ägyptischen Truppen.

\section{B. Das Eherecht der peregrinen Soldaten.}

\section{Papyrus Cattaoui Col. III.}

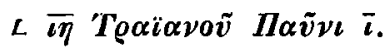

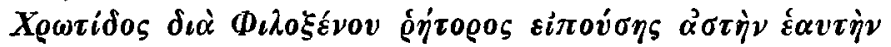

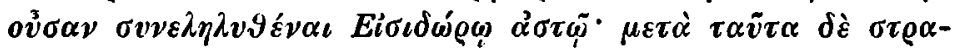

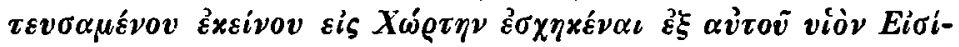

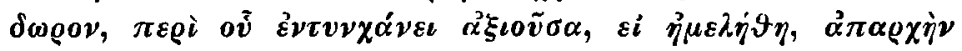

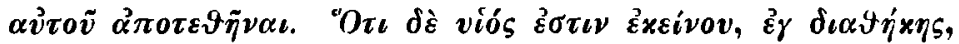

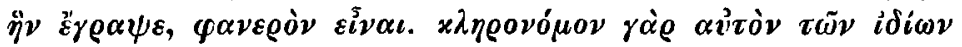

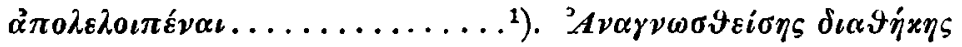

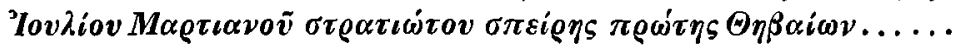

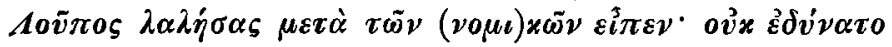

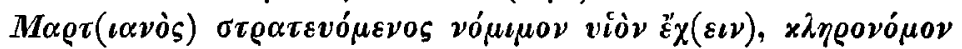

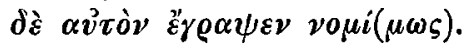

\section{Uebersetzung:}

Im 18. Jahr des Trajan am 10. Payni.

Khrôtis giebt durch ihren Vertreter Philoxenus an, dass sie, eine Alexandrinerin, eine Verbindung eingegangen sei mit dem Alexandriner Isidorus. Nachdem jener darauf Soldat geworden und in Khortis stationirt sei, habe sie ihm einen Sohn Isidorus geboren. In Betreff desselben stelle sie den Antrag, die vicesima hereditatium, wenn es unterlassen sein sollte, zu

1) Ich bin auf die von Botti mitgetheilten Bruchstücke der Urkunde angewiesen. 
hinterlegen(?). Dass er aber der Sohn des Isidorus sei, werde offenkundig aus dem von diesem hinterlassenen Testamente; denn in demselben habe er ihn zum Erben seines Vermögens eingesetzt........ Nach Verlesung des Testamentes des Iulius Martianus, Soldaten der cohors 1. Thebaeorum, entscheidet Lupus nach Besprechung mit den rechtskundigen Beisitzern: Martianus konnte während seiner Dienstzeit keinen legitimen Sohn erhalten; vollkommen rechtmässig aber hat er denselben im Testament zum Erben eingesetzt.

Es handelt sich um eine Entscheidung des oben (S.50) genannten praef. Aeg. M. Rutilius Lupus, die datirt ist vom 4. Juni 115.

Isidorus und Khrôtis leben zusammen in einem matrimonium Alexandrinum, müssen sich aber trennen, als Ersterer als Soldat in die an der nubischen Grenze stationirte cohors I. Thebaeorum eintritt. Als solcher erhält er den Namen Iulius Martianus (Isidorus), ohne aber mit der römischen Nomenclatur auch zugleich das römische Bürgerrecht zu erbalten. Bald darauf wird ihm ein Sohn Isidorus geboren, der schon vor seinem Diensteintritt concipirt ist. Iulius Martianus stirbt als Soldat und setzt in seinem Testamente den Isidorus zum Erben ein. Die Mutter stellt auf Grund dieser testamentarischen Erbeseinsetzung ihres Sohnes den Antrag, denselben als eheliches Kind und demzufolge heres suus et legitimus des Vaters von der vicesima hereditatium zu entbinden. Der Richter aber entscheidet:

Auch das matrimonium Alexandrinum eines Soldaten ist während der Dienstzeit suspendirt; ein vor derselben concipirtes, während derselben geborenes Kind ist illegitim. Anders verhält es sich dagegen mit der testamentarischen Erbeseinsetzung: Martianus konnte jeden extraneus zum Erben einsetzen. - Ueber die Zahlung der vicesima als selbstverständliche äussert sich der Richter nicht.

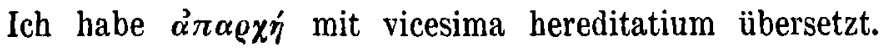
Das Wort bedeutet ursprünglich „Erstlingsgabe", wird dann aber folgerichtig auch von einer ,bestimmten Quote" gebraucht, die von einem Vermögen, von Beute, von einem Zehnten, von Früchten zu entrichten ist. So finden wir es C. I. Att. I, 257 
(s. Böckh, Staatshaushalt der Athener, 2. Aufl. II p. 376, 381); C.I.Gr. II, 2855, 13; Platon. Nóno 7 p. 806 D. Im Pap. Taurin. I p. 7 lin. $8 \mathrm{sqq}$. hat es in diesem Sinne die unzweifelhafte Bedeutung von einer dem Fiscus zu zahlenden Erbschafts-

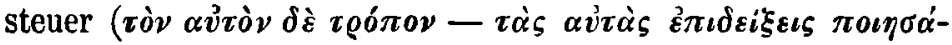

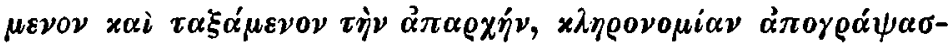

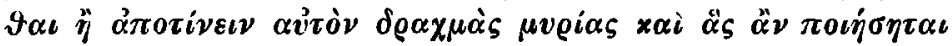

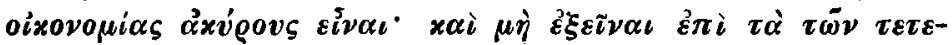
$\lambda \varepsilon v \tau \eta x o ́ \tau \omega \nu ~ \varepsilon ̇ \pi \iota \pi \tau \rho อ \varepsilon ์ \varepsilon \sigma \vartheta \alpha \iota$ [s. C.P.R. I, 18, 33; U.B.M. 388 II, 12;448; Mnemosyne $25 \mathrm{II}, 173 \mathrm{sqq}$.$] ). Und so ist das$ Wort auch hier aufzufassen (ebenso wie U.B.M. 30: $\eta^{i} \dot{\alpha} \pi \alpha \varrho x \grave{\eta}$

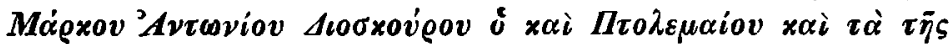
$\mu \eta \tau \varrho \grave{s} \varsigma \alpha \dot{v} \tau o \bar{v})$. Dass sonst in ägyptischen Urkunden direct

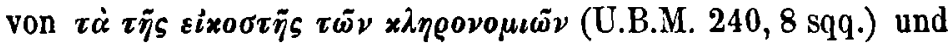

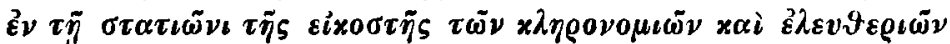

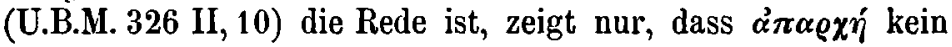
officieller Ausdruck für diese Erbschaftssteuer ist.

Isidorus erhält bei seinem Eintritt in die cohors I. Thebaeorum zwar römische Nomenclatur, aber nicht Civität; dies ist in dieser Zeit nichts Auffallendes. Der Behauptung Wilckens (Hermes 1892, 290), dass, wenn wir einen Peregrinen mit römischem Gentilnamen antreffen, wir anzunehmen berechtigt sind, einen Peregrinen mit römischer Civität vor uns zu haben, kann ich nicht zustimmen. Hierfür einige Belege: In der U.B.M. 423 (wohl aus dem Anfang des 2. Jahrhunderts) haben wir den Brief eines Aegypters Apion an seinen Vater vor uns, der ausgehoben, nach Misenum befördert wird und von dort, mit einem viaticum versehen, zu seinem Truppentheil kommt. Bei seinem Diensteintritt erhält er römische Nomenclatur (v. 22:

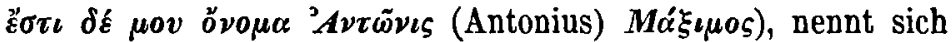
selbst in dem Briefe mit seinem alten Namen 'A $A \pi{ }^{\prime} \omega \nu$ (v. 1 in verso), ohne dass von der Ertheilung der civitas R. die Rede ist. In der von Wilcken l.l. angeführten U.B.M. 655 aus dem

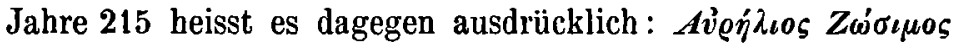

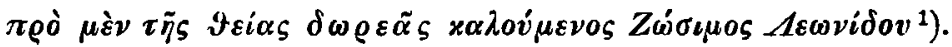

Viele der in den Militärdiplomen genannten Soldaten mit italisch geformtem Namen sind Nichtbürger (z. B. D. 47 [aus

1) Derselbe wird wohl U.B.M. 222, ã (a. d. J. 213/14); 64, 2 (a. d. J. 215/17); ev. auch U.B.M. 97, 6 u. 15 (a. d. J. 201/202) genannt. 
dem Jahre 133]: Claudio Motti f. Novano Helvetio; D. 51 [aus dem Jahre 138]: Sex. Iulio Primi f. Primo 'Treviro; D. 76 [aus dem Jahre 178]: Valerio Valeri f. Valenti castr[is]).

Ganz ausdrücklich wird der Soldat Octavius Valens, dessen Frau Cassia Secunda heisst, im Papyr. Catt. Col. IV aus dem Jahre 142 (s. S. 65 ff.) civis Alexandrinus genannt.

Ich will nur endlich noch auf die italische Namensform der classiarii und equites singulares verweisen, die auch nach Caracalla nur selten die Civität erhielten.

So glaube ich, dass das Verbot des Claudius (Suet. Claud. 25: peregrinae condicionis homines vetuit usurpare nomina dumtaxat gentilicia) nur auf seine Zeit zu beziehen und sodann allgemein zu verstehen ist. Soldaten werden immer Ausnahmen gebildet haben, indem sie, auch ohne die Civität zu erhalten, bei ihrem Diensteintritt das gentile des Kaisers (oder auch früherer Kaiser) oder des Beamten resp. Commandanten, unter dem sie ausgemustert wurden (so in Aegypten des praef. Aeg.: U.B.M. 447; Eph. ep. VII p. 456 sqq., I, 20-24), erhielten.

Das matrimonium Alexandrinum bleibt also in unserem Fall auch während der Dienstzeit bestehen, wird aber von dem Militärgesetz ignorirt und als suspendirt betrachtet. Während im Allgemeinen das matrimonium peregrinum der Soldaten in trajanischer Zeit noch rechtlich anerkannt ist, gilt die alexandrinisch-griechische Ehe als suspendirt. Wie die griechische Stadtgemeinde, so wird auch die griechische Ehe und ebenso das griechische Testament den gleichartigen römischen Institutionen analog behandelt.

Der Sohn der Khrôtis ist kein legitimes Kind des civis Alexandrinus Iulius Martianus Isidorus, aber vollkommen rechtmässig von ihm als extraneus zum testamentarischen Erben eingesetzt. Als civis Alexandrinus aber, der ebensowenig wie der Römer von der vicesima befreit ist, und als extraneus hat er die Erbschaftssteuer zu zahlen.

Soweit entspricht das alexandrinische dem römischen Recht. In einer Hinsicht ist aus unserer Urkunde ein Unterschied zwischen beiden zu constatiren: Das Kind Isidorus ist vor dem Eintritt des Vaters in das Heer concipirt, während der Dienstzeit geboren. Zwischen dem Zeitpunkt der Conception und der Geburt ist das matrimonium iustum iuris Alexandrini 
nach römischem Militärgesetz in eine illegitime Verbindung verwandelt; das Kind ist also legitime conceptus, illegitime natus. In Bezug auf Fälle, wo der status der Eltern resp. der Mutter in dieser Zeit sich verändert, stellt Gaius $(I, 89)$ die Regel auf: hi qui illegitime concipiuntur, statum sumunt ex eo tempore quo nascuntur - at hi qui legitime concipiuntur, ex conceptionis tempore statum sumunt. Dehnen wir diesen Grundsatz auch auf Veränderungen hinsichtlich der rechtlichen Natur der geschlechtlichen Verbindungen aus, dann finden wir in unserer Urkunde eine Abweichung von diesem Princip, die wir nur auf das alexandrinische Recht zurückführen können. Dieses hat scheinbar den Satz aufgestellt: Das Massgebende für die Legitimität der Kinder, ob sie ehelicher oder unehelicher Conception sind, ist die Beschaffenheit der geschlechtlichen Verbindung zur Zeit der Geburt ${ }^{1}$ ).

2. U.B.M. 114 Col. I v. 14 sqq., Col. II.

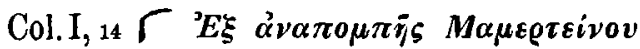

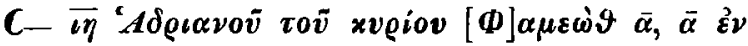

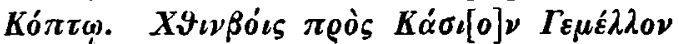

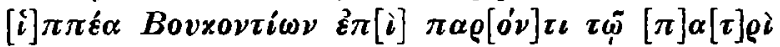

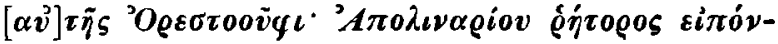

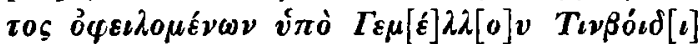

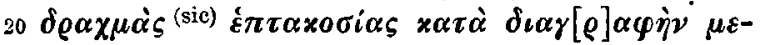

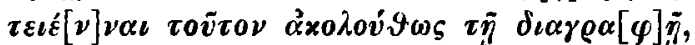

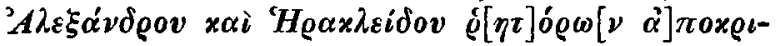

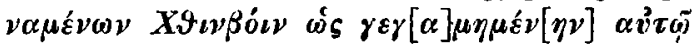

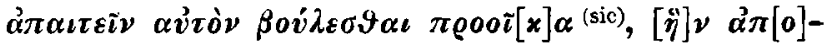

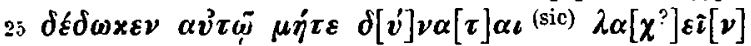

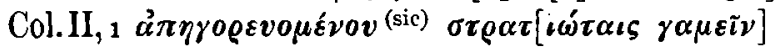

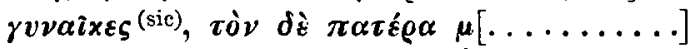

(sic)

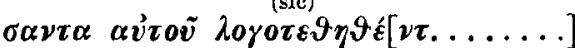

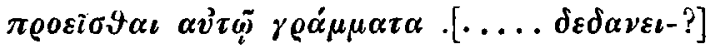

1) Bottis Bemerkungen zu dieser Urkunde: „cosi il povero Isidoro non ebbe per diritto di nascita la cittadinanza alessandrina (s. hierzu Pap. Cattaoui Col. IV, S. 67); Grotis passò per concubina; il di lei figlio fu tuttavia erede legittimo perchè il matrimonio illegale fu equiparato

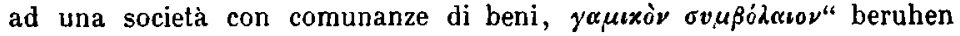
auf geringem Verständniss des Inhaltes. 


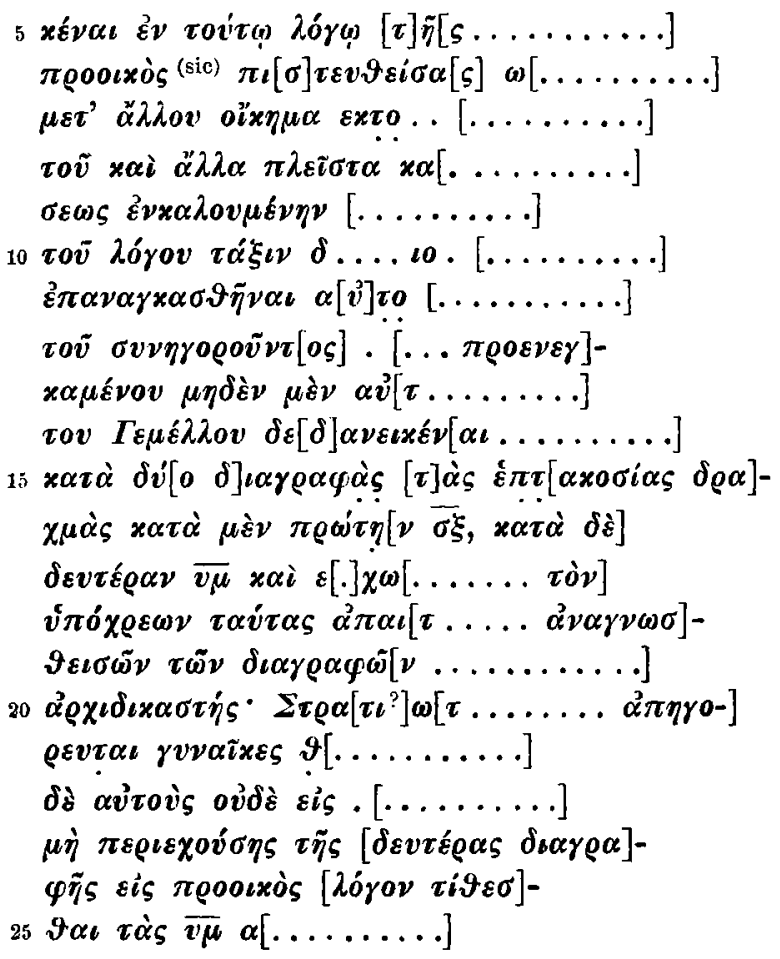

Uebersetzung:

„Entscheidung in Folge Delegation durch Mamertinus im 18. Jahre unseres Herrn Hadrian am 1. Phame(n)oth, Seite 1 (des Journals), in Coptus.

Klage der Chthinbois gegen Cassius Gemellus, Reiter in der ala Vocontiorum, vorgebracht unter Assistenz ihres Vaters Orestouphi.

Apol(1)inaris, Vertreter (der Klägerin), behauptet, Gemellus schulde der Chthinbois 700 Drachmen laut Schuldverschreibung; diese fordere sie von ihm gemäss derselben. Darauf antworten Alexander und Heraclides als Vertreter (des Beklagten): Chthinbois wolle von Gemellus, als ob sie seine Ehefrau wäre, die Mitgift fordern, die sie ihm zurückgegeben habe und nicht bekommen könne, da es ja den Soldaten verboten sei, Ehefrauen zu nehmen" u. s. w.

Der Rest der 2. Columne ist so lückenhaft, dass eine zusammenhängende Uebersetzung nicht möglich ist. 
Folgendes scheint mir der Inhalt der Reden und Gegenreden der Vertreter der Klägerin und des Beklagten, sowie der Entscheidung des Richters zu sein:

Klägerin: Gemellus schuldet mir 700 Drachmen auf Grund einer Schuldverschreibung (I, 18-21).

Beklagter: Es handelt sich nicht um ein Darlehn, wie Klägerin angiebt, sondern um eine $\sigma v \gamma \gamma \varrho \alpha \varphi \grave{\eta} \delta \alpha \nu \varepsilon i o v$ (s. Mitteis, Reichsrecht S. $468 \mathrm{ff}$; s. vorher S. 52 f.), d. h. um ein verschleiertes Brautgeschenk des Mannes an die Frau. Als Soldat konnte dieser aber keine giltige Ehe eingehen; der fingirte Darlehnsvertrag ist also nichtig (I, 22-25; II, 1-6) ${ }^{1}$ ).

Klägerin: Es kommt nicht eine, sondern zwei Schuldurkunden in Betracht ${ }^{2}$ ): in der ersten handelt es sich um ein „Darlehn" von 260, in der zweiten um ein solches von 440 Drachmen. Wenn auch der erste Vertrag ein verschleierter Dotalvertrag ist, der zweite bezieht sich auf ein wirklich hingegebenes Darlehn. Ich verlange daher wenigstens die 440 Drachmen (II, v. 14-18).

Der Richter lässt die beiden Schuldurkunden verlesen

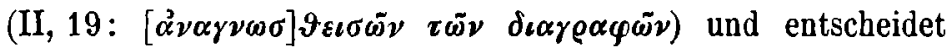
dann:

Die Soldaten haben kein Eherecht, sie dürfen daher auch keine Ehecontracte abschliessen (II, 20-22). Die erste Urkunde ist ein solcher; aus der zweiten aber geht nicht hervor, dass sie ein verschleiertes Brautgeschenk enthält (II, 23-25):

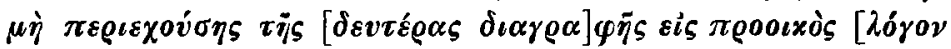
$\tau i \vartheta \varepsilon \sigma] \vartheta \alpha \boldsymbol{\tau} \dot{\alpha} \varsigma \overline{v \mu}(\delta \rho \alpha \chi \mu \dot{\alpha} \varsigma)-)$.

440 Drachmen.]

[Ich verurtheile daher den Gemellus zur Zahlung von

Die Entscheidung ist vom 25. Februar 134 datirt. In der uns vorliegenden Gestalt repräsentirt unsere Urkunde einen Auszug aus dem Tagebuch eines $\alpha^{2} \varrho x \imath \_x \alpha \sigma \tau \dot{\eta}^{\prime} \varsigma$ und ist, wie wir S. 52 bemerkt haben, mit analogen Entscheidungen zu bestimmtem Zwecke nach dem genannten Jahre zusammengestellt worden.

Der $\alpha \varrho x \iota \delta\llcorner x \alpha \sigma x \dot{\eta} \varsigma$ wird von Strabon $(17,1,12$ p. 797) unter den städtischen Beamten in Alexandria genaunt. Mindestens seit Trajan ist er aber zugleich Unterbeamter des Statthalters,

2) v. 7-13 sind in Folge der grossen Lücken unklar. - 2) Wie auch im Papyr. Catt. Col. I. 
fungirt auch ausserhalb Alexandrias und für die ganze $\chi \omega$ œ́a und hat Ritterrang (U.B.M. 73; $136 ; 231 ; 241 ; 388 ; 578 ; 614$; Papyr. Catt. Col. I; Papyr. Berol. Bibl. ed. Parthey n. 8; Pap. Brit. Mus. $711=$ Grenfell and Hunt, Greek Papyri II n. 71). Meistens sind mit seinem Amte, wie auch mit dem anderer ägyptischer Beamten, religiöse Functionen verbunden (s. Mommsen in dieser Zeitschr. (XVI) 1895, S. 190 A. 1; s. auch U.B.M. 455; 578, 7 u. $9 ; 614,7$ u. 10$)$.

Dem $a^{3} \varrho \chi \imath \delta \iota x \alpha \sigma \tau \eta \zeta$ wird die Rechtsprechung von dem praef. Aeg. Sex. Petronius Mamertinus (s. Hermes XXXII, 219 f.) delegirt. Wir kennen aus den ägyptischen Urkunden noch einen anderen Fall einer Delegation von demselben Präfecten an

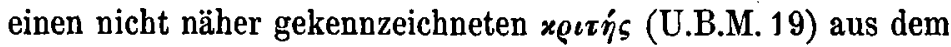
Jahre 135 (s. Wilcken, Philologus 53 (1894), 107; Mommsen, in dieser Zeitschr. XIV (1893), S. 5). Mommsen hat die technische Bedeutung von $\alpha^{\prime} \nu \alpha \pi \hat{\varepsilon} \mu \pi \varepsilon \iota \nu, \alpha^{3} \nu \alpha \pi \rho \mu \pi \dot{\eta}$, wonach der competente Magistrat nach Belieben einen iudex datus (sei es nun städtische Beamte, sei es Officiere) bestellen konnte (in dieser Zeitschr. XII (1892), S. $292 \mathrm{f}$.) im Anschluss an die Worte des Papyrus Rainer 1492 (= Wessely l.1. n. 18) eingehend erörtert.

Das Urtheil ergeht in dem u. A. durch den laterculus militum aus augustischer Zeit (C.I.L. III, $6627=$ Eph. ep. V, 15) und neuerdings besonders durch die Ausgrabuugen und Funde von Flinders Petrie (Coptos 1896; s. bes. p. 26 sqq. [Hogarth]) bekannten Coptos.

Der eques alae Vocontiorum (s. auch Eph. ep.VII p. 426 sqq. n. 9 aus domitianischer Zeit, in den oberägyptischen Bergwerken gefunden) Cassius Gemellus, als dessen Frau die Aegypterin Chthinbois genannt wird, hat beim Eintritt in das Heer zwar römische Nomenclatur erhalten, nicht aber die Civität (s. S. 59 f.).

Ein peregriner Soldat ist also während seiner Dienstzeit mit einer peregrina eine Verbindung eingegangen, die vollkommen den vom ägyptischen Recht verlangten Erfordernissen

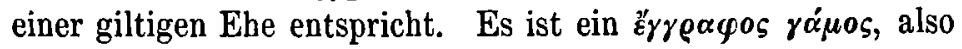
ein matrimonium iustum iuris peregrini, das nach unserer bisherigen Annahme während der Dienstzeit nicht verboten war. Der Wortlaut unserer Urkunde aber (I, 23-25; II, 1) zeigt deutlich, dass im Jahre 134 das Eheverbot auch auf die Ehen 
peregriner Soldaten übertragen war. Diese grundlegende Veränderung werden wir, wie bei der zusammenfassenden Darlegung des Eherechtes der peregrinen Soldaten (S. 68 ff.) auszuführen, seit dem Beginn der Regierung des Hadrian constatiren können.

Die Forderung von 700 Drachmen wird von der beklagten Partei mit der Motivirung bekämpft, dass es sich hier um eine $\sigma v \gamma \gamma \varrho \alpha \varphi \grave{\eta} \delta \alpha \nu \varepsilon i o v$, die einen integrirenden Bestandtheil eines von einem Soldaten abgeschlossenen Ehecontractes bildet, handle. Im Gegensatz zu der im Allgemeinen üblichen Praxis der römischen Gerichte in Aegypten schliesst sich der Richter der Behauptung an, dass ein solcher Ehecontract, da er eben von einem Soldaten abgeschlossen, nichtig sei. Damit hält er strenge am Gesetze fest; die militärische Disciplin darf nicht verletzt werden. Trotzdem aber findet er eine Handhabe, um der verlassenen Frau zu ihrem Rechte gegenüber dem sauberen Gatten $\mathrm{zu}$ verhelfen. Es ist, möchte man sagen, eine ritterliche Empfindung, welche den Richter bei seiner Entscheidung leitet:

Erst hat Gemellus, des Verbotes sich wohl bewusst, als Soldat eine peregrine Ehe geschlossen und, um die Militärgesetze zu umgehen, einen fingirten Vermögensvertrag abgeschlossen. Jetzt, wo er von der treulos verlassenen Frau auf Herausgabe der versprochenen Geldsumme verklagt wird, kehrt er in frivoler Weise den Spiess um und beruft sich auf jene Gesetze. „Von einer Ehe konnte ja nicht die Rede sein; darum brauche ich mein gegebenes Wort nicht zu halten", so argumentirt er. Der Richter aber lässt ihm eine gerechte Strafe $\mathrm{zu}$ Theil werden, indem er den zweiten Vertrag für einen wahren Darlehnsvertrag erklärt und ihn zur Zahlung von 440 Drachmen verurtheilt.

Es ist wohl kein Zufall, dass die beiden der „Frau" eines Soldaten günstigen Entscheidungen (s. U.B.M. 114 I, v. 5-13: S. 51 ff.) auf einem Papyrus vereinigt sind. Damit dürfte sich die Frage nach dem Grunde dieser Vereinigung erledigen.

$$
\text { Pap. Cattaoui Col. IV. }
$$

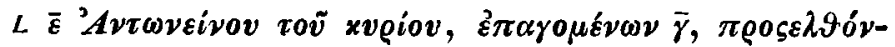

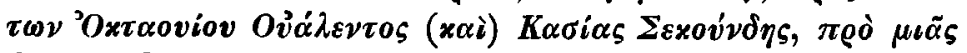

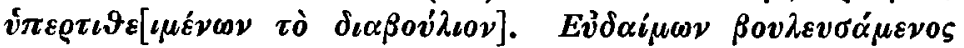

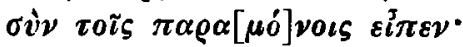

Zeltschrlft für Rechtsgeschichte, XVIII. Rom. Abth. 


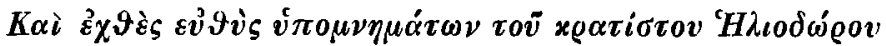

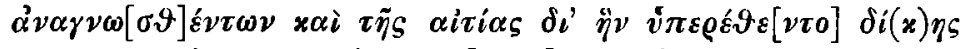

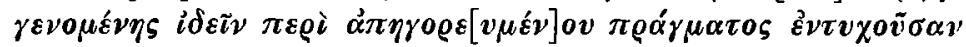

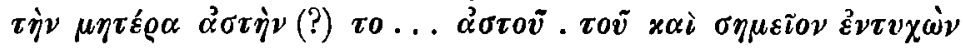

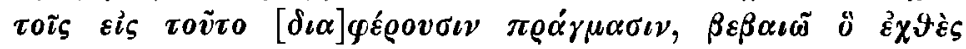

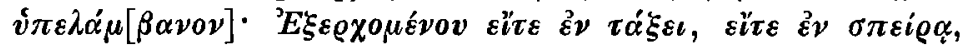

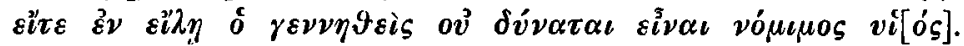

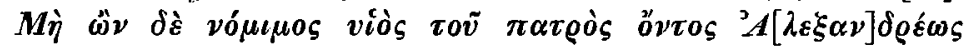

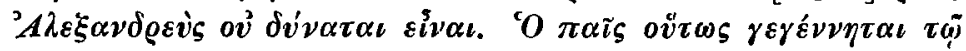

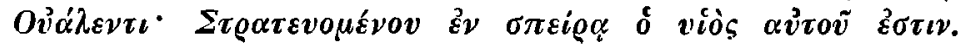

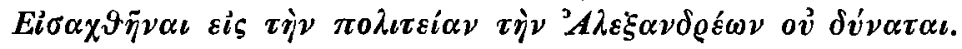

Wir haben hier das Enderkenntniss in einer „Ehesache“ eines miles civis Alexandrinus vor uns. Die Entscheidung wird am 26. August 142 von dem in Alexandria amtirenden idiologos Eudaemon gefällt, dessen frühere Carriere wir nach der sehr wahrscheinlichen Vermuthung Hirschfelds (bei Friedländer, Sittengesch. $\mathrm{I}^{6}, 186$; s, auch Jung, Wiener Studien 1892, 256) durch zwei Inschriften (Bull. d. corr. hell. III p. $257=$ C.I.L. III, 7116; C.I.L. III, 431) kennen lernen (s. vit. Hadr. 15).

Die Verhandlung war am 25. August ausgesetzt und auf den nächsten Tag vertagt; an diesem wird die Entscheidung gefällt, die das bedingt abgegebene Gutachten des ersten Verhandlungstages bestätigt.

In der U.B.M. 19 (bes. I, 5 u. 19) setzt der vom praef. Aeg. delegirte iudex die Entscheidung aus bis nach erlangter Instruction seines Mandanten, da es sich um eine Rechtsfrage (s. Ulpian. D. 5, 1, 79, 1) handelt (Mommsen in dieser Zeitschr. XIV (1893), 1 ff.). Nachdem die Belehrung erfolgt, wird das Urtheil gefällt.

Auch in unserem Fall handelt es sich um eine Rechtsfrage: um die Legitimität (und im Anschluss daran um den status) der in einer alexandrinischen Soldatenehe geborenen Kinder. Instruction ist aber nicht mehr nöthig. Die Rechtsfrage ist schon durch dem Richter vorliegende Rescripte ( $\boldsymbol{v} \pi \boldsymbol{r}$ $\mu \nu \eta_{\mu \alpha \tau \alpha}$; s. Wilcken, Philologus 53 (1894), 103; s. auch U.B.M. $613,18$ u. $27 / 28 ; 614,7)$ des praef. Aeg. C. Avidius Heliodorus (der von 138 - c. 147 amtirte; s. Hermes XXXII, 220 ff.) entschieden. Sie werden am Beginn des ersten Verhandlungstages 
verlesen; die Vertagung findet nur zum Zwecke der Erhebung des Beweises statt. Das Urtheil wird dann am zweiten Tage auf Grund der allgemeinen Entscheidungen des Präfecten gefällt (vgl. auch U.B.M. 613 , v. 19; 27/28).

Dieser hat in derselben zwei Sätze aufgestellt:

1. auf Grund der römischen Militärgesetze: Die Soldaten aller Truppengattungen des Landheeres (Legion, Cohorte, Ala), d. h. also Bürger- und peregrine Soldaten, haben kein Eherecht; ihre während der Dienstzeit geborenen Kinder sind illegitim.

2. auf Grund des alexandrinischen Rechtes: Der illegitime Sohn eines civis Alexandrinus kann nicht die Civität seines Vaters erlangen.

Daraufhin entscheidet Eudaemon in unserem speciellen Falle: Aus der Verbindung zwischen dem cohortalis Octavius Valens, dessen civitas Alexandrina feststeht, und der Alexandrinerin Cassia Secunda ist während der Dienstzeit ein Sohn entsprossen. Das an und für sich vollgiltige matrimonium Alexandrinum gilt vor dem Militärgesetz als nicht existent (s. Pap. Cattaoui Col. III: S. 60), der Sohn daher als illegitim. Er kann daher auch nicht den status seiner Eltern (die civitas Alexandrina) erlangen.

In der Urkunde des Papyrus Cattaoui aus dem Jahre 115 (Col. III) war von einer Entziehung der civitas Alexandrina gegenüber dem in legitimer Verbindung (matrimonium iustum Alexandrinum) concipirten, in illegitimer (während der Dienstzeit) geborenen Kinde nicht die Rede. In der Col. IV wird ausdrücklich der Mangel desselben betont.

Diese Urkunde aus dem Jahre 142 lehrt uns also erstlich eine wohl erst im 2. Jahrhundert unter römischem Einflusse ausgebildeteEigenthümlichkeit des alexandrinischen ${ }^{1}$ ) Rechts kennen, sodann aber spricht sie offen und klar das mangelnde Eherecht für alle Soldaten des Landheeres aus: Nicht nur die römische und alexandrinische, sondern auch jede peregrine Ehe - das besagen ihre Worte - ist während der Dienstzeit suspendirt.

1) Im griechischen Recht (so besonders im attischen) bestand kein Hinderungsgrund, die vó Bürgerverband einzuführen. Sie nehmen oftmals eine niederere Stellung als die Vollbürger ein, haben aber immer Antheil an der nodızia (Hermann, Lehrb. der griech. Staatsalterthümer, 6. Aufl. (1892), S. 445 u. 449). 
Die drei zuletzt besprochenen Urkunden stossen die bisherigen Ansichten über "das Eherecht der peregrinen Soldaten" in vielen wesentlichen Punkten um. Es dürfte daher nicht werthlos sein, die Entwickelung desselben in den ersten Jahrhunderten der Kaiserzeit an der Hand der alten und neuen Documente zusammenfassend zu beleuchten.

Die leges Iuliae et Papia Poppaea (= vó $\mu$ : : Dio 60, 24) sprechen das Eheverbot für Soldaten aus. $\mathrm{Da}$ sie ius civile sind, umfassen sie nur das matrimonium (iustum) iuris civilis, nicht dagegen das matrimonium iniustum und das matrimonium iuris peregrini.

Eine Verbindung zwischen cives $R$. und peregrinae ohne conubium ist niemals eine vollgiltige Ehe, nur "Halbehe", matrimonium iniustum, non legitimum (Concub. 58), contubernium (Concub. 32 A. 61). Erst im 3. Jahrhundert wird diese Halbehe dem matrimonium iustum gleichgestellt, alle Vortheile und Nachtheile der "leges" auf sie übertragen (Concub. 59). Vor dem 3. Jahrhundert existirt also für cives $R$. juristisch kein matrimonium iniustum; ein specielles Verbot für Soldaten ist nicht nothwendig. Es steht auf derselben rechtlichen Stufe wie der Concubinat eines civis mit einer civis $R^{1}{ }^{1}$ ): wie uns U.B.M. 114 I v. 14 sqq. zeigt, kann eine solche Verbindung von einem Soldaten jeder Zeit gelöst werden, hat keine bindende Kraft. Concubinat wie Halbehe geniessen die gleiche factische Duldung ohne gesetzliche Sanction (s. Conc. 102, 109, 111). Dies.

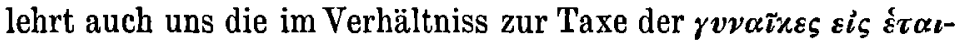

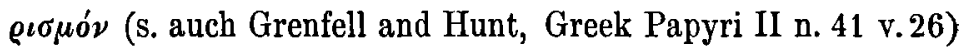

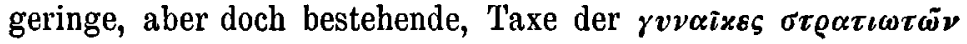

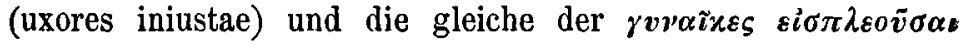
(women immigrant $=$ those coming voluntarily and not under

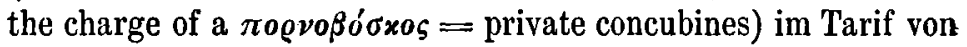
Coptos (Hogarth bei Flinders Petrie Coptos p. 27 sqq.; s. S. 64).

2) Hierdurch werden die Bemerkungen Küblers in der Besprechung meines „Concubinates“ in dieser Zeitschr. XVII hinfällig: „Wenn man den Soldaten die Ehe überhaupt einmal gestattet, warum sollte man ihnen dann das iustum matrimonium verwehren ?" (S. 362). - „In dem Augenblicke, wo ihm verstattet wurde, mit einer Frau zusammenzuwohnen, fiel jeder Grund fort, ihm das justum matrimonium zu verbieten" (S. 364). 
Ebensowenig ist natürich ein Verbot des matrimonium iuris peregrini in den "leges" enthalten. Im Gegensatz zu jener "Halbehe" gilt dieses aber vor dem Militärgesetz als vollgiltige peregrine Ehe. Es ist den peregrinen Soldaten nicht nur factisch erlaubt ${ }^{1}$ ), es werden auch rechtliche Wirkungen an dasselbe geknüpft. Die Soldaten erhalten nach ihrer Entlassung (civitas für sich, ihre Frau und Nachkommen und) conubium mit rückwirkender Kraft: die vollgiltige peregrine Ehe verwandelt sich nach der Entlassung in ein vollgiltiges matrimonium iuris civilis, und zwar gilt sie als solches vom Augenblick ihrer Eingehung $a b^{2}$ ).

Eine Ausnahme von dieser rechtlichen Anerkennung der peregrinen Soldatenehe macht dagegen die Ehe der milites cives Alexandrini und wohl allgemein die griechische Soldatenehe. Das matrimonium iustum iuris Alexandrini wird während der Dienstzeit suspendirt: s. Papyrus Cattaoui Col. III (s. S. 58 ff.) und IV (s. S. 66 f.). Der aus solcher jetzt illegitimen Verbindung von cives Alexandrini geborene Sohn ist nicht nur illegitim, sondern erhält (wohl erst seit Hadrian) auch nicht einmal die civitas Alexandrina (Pap. Catt. Col. IV: S.67).

Die Suspension des matr. Alexandrinum bildet die Vorstufe zur allgemeinen Suspension des gemeinen matrimonium iuris peregrini der Auxiliare während der Dienstzeit.

Diese Aenderung in Bezug auf das peregrine Militär-Eherecht ist zurückzuführen auf den Beginn der Regierung Hadrians. Nach dem übermässig soldatenfreundlichen Trajan (s. S. 55) tritt sofort eine Reaction unter seinem Nachfolger ein.

Das matrimonium der nach dem Jahre 117 neu eingetretenen peregrinen Auxiliare wird von den römischen Militärgesetzen ebenso behandelt wie das matrimonium iniustum der milites cives R. Es existirt kein directes Verbot, das Gesetz ignorirt aber eine solche Verbindung, die keine rechtliche Wirkung und Bindung mehr erzeugt (U.B.M. 114 Col. I, v. 14 sqq.: S. 64 f.; Pap. Cattaoui Col. IV: S. 67).

Die vor dem Regierungsantritt Hadrians ins Heer einge-

2) Es ist von den uxores der milites peregrini die Rede (s. Militärdiplom 3 (a. d. J. 65); 23 (a. d. J. 93); 35 (J. 107); 39 (J.114); 52 (vor 142). - 2) Militärdiplom 3; 35; 37 (J.110); 39; 31 (J. 99); 46 (J. 129); 47 (J. 133); 48 (J. 134); 51 (J. 138); 52; U.B.M. 113 (J. 143). 
tretenen Auxiliare behalten während ihrer Dienstzeit die alten Eheprivilegien. Die 1.Generation, welche derselben beraubt ist, sind die im Jahre 142 (nach 25jähriger Dienstzeit) entlassenen Veteranen. Diesem Jahrgang begegnen wir U.B.M. 113 : die Urkunde giebt einen Auszug aus den Acten des praef. Aeg. C. Avidius Heliodorus über die vom 15. Februar bis 16. Mai 143 unter seinem Vorsitz abgehaltene ż $\pi i x \rho \iota \sigma \varsigma$ (s. meinen Aufsatz Philologus LVI, $206 \mathrm{ff}$.). Es erscheinen hier neben einander

1. Veteranen, welche die vollen Privilegien bei ihrer Entlassung erhalten haben (S. 69),

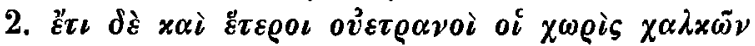

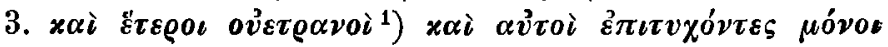

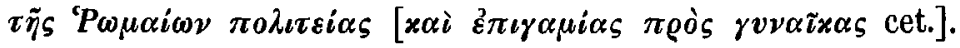

Also neben den mit Vollprivilegien Entlassenen finden wir noch zwei Kategorien: 2. oi $\chi \omega$ gis $\chi \alpha \lambda x \tilde{\omega} \nu=$ sine aere, $d . \mathbf{b}$. die ohne Diplom ${ }^{2}$ ) Entlassenen, in Folge von missio ignominiosa oder causaria. 3. Die im Besitz eines nur beschränkte Privilegien enthaltenden Diploms befindlichen Veteranen (s. Conc.120): Das ist der Jahrgang 117.

Die Veränderung hinsichtlich des Eherechtes der peregrinen Auxiliare übt demnach auch ibren Einfluss aus auf die ihnen nach der Entlassung zuertheilten Privilegien. Eine gesetzlich während der Dienstzeit ignorirte Verbindung soll auch nach derselben in Bezug auf die Vergangenheit keine nachträgliche Sanction erhalten, sie bekommt eine solche und rechtliche Wirkungen nur für die Zukunft. Die auxiliarii erhalten jetzt nur noch civitas für sich (und ihre Frau); ibr während der Dienstzeit von dem Militärgesetz ignorirtes matrimonium peregrinum wird in ein matrimonium iustum iuris civilis verwandelt, jedoch ohne rückwirkende Kraft; die während der Dienstzeit geborenen Kinder bleiben peregrini und illegitim.

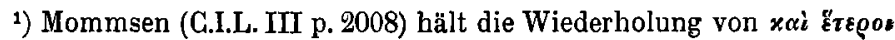

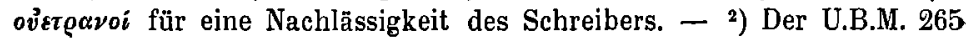
genannte Veteran hinterlegt beim trib. mil. leg. II. Traianae Fortis sein Diplom, das in Bezug auf seine äussere Form als $\delta \dot{z} \lambda \tau_{0} \chi \alpha \lambda x \dot{\eta} \dot{\varepsilon} x \sigma q \varrho \alpha-$ $\gamma \iota \sigma \mu \varepsilon \dot{\varepsilon} \eta$ (v. 21), in Bezug auf seinen Inhalt als $\delta \iota x \alpha i \omega \mu \alpha$ (v. 17) bezeichnet

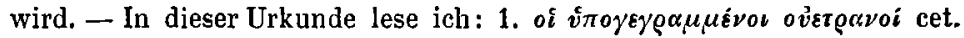

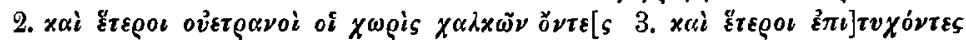

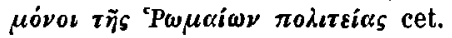


dus dieser veränderten eherechtlichen Qualification der Auxiliare ergiebt sich naturgemäss die Uebertragung des Ersatzinstitutes der ex castris auch auf das ius peregrinum. Das Institut war am Anfang der Kaiserzeit ins Leben gerufen (auf Grund der eigenartigen ägyptischen Verhältnisse), um einen festen Soldatenstamm zu schaffen, und gewährte den im matrimonium iniustum (mit peregrinae) der Legionare geborenen Kindern die Civität (Conc. 111). Jetzt, wo auch die peregrine Soldatenehe für illegitim erklärt wird, soll den auch nach der Dienstzeit nicht legitimirten Kindern eine gewisse Halblegitimität als ex castris ertheilt werden. Wiederum wird dieses Ersatzinstitut zuerst in Aegypten zur Anwendung gebracht sein (als Ersatz für das suspendirte matrimonium Alexandrinum). Während aber die Einrichtung in ihrer ursprünglichen Gestalt während des ganzen 1. Jahrhunderts fast allein auf Aegypten beschränkt blieb - in Afrika hatte es noch unter Trajan kaum Wurzel gefasst (Recueil de la société de Constantine 1882 378 sq.; Mommsen, Hermes 19,8) -, scheint sie in ihrer Neuanwendung auf das ius peregrinum alle Provinzen gleichmässig betroffen zu haben.

Das älteste uns bekannte Document eines solchen peregrinen ex castris weist auf einen Soldaten, der im Beginn der Regierung des Hadrian ins Heer eingetreten ist: es ist das Militärdiplom eines eques cohortis V. Lucensium et Callaecorum aus den Jahren 142-145 ${ }^{1}$ ) (C.I.L. III p. 1984, D. 59). Ausser diesem besitzen wir nur noch ein anderes Diplom aus dem Jahre 178 (C.I.L. III p. 1993, D. 76 : gefunden in Tirnowa).

So bilden also Aufhebung des Eherechtes der peregrinen Auxiliare, Einschränkung der ihnen bei der Entlassung ertheilten Privilegien, Uebertragung des Instituts der ex castris, auf das ius peregrinum die einzelnen Glieder derselben Entwickelung.

In Gegensatz hierzu bleiben für die Flottens oldaten und equites singulares die eherechtlichen Verhältnisse, die nach der Entlassung gewährten Privilegien auch nach dem

1) Die Formel civitatem R., qui eorum non haberent, dedit ... weist auf die Zeit seit 142; die Distributivzahl bei der Angabe der Dienstjahre auf die Jahre vor 146. Das Diplom stammt demnach aus den Jahren 142 bis 145. Der Soldat ist in den Jahren 117-120 in das Heer eingetreten. 
Jahre 142 dieselben. Ihre Ehe erhält während der Dienstzeit die Sanction ${ }^{1}$ ) der Militärgesetze, wird nach derselben in ein iustum matrimonium iuris civilis mit rückwirkender Kraft (in Hinsicht auf die vorher geborenen Kinder) verwandelt (Conc. 119; $95 \mathrm{ff}$.).

Der Grund für diese verschiedene Behandlung ist in der abweichenden staatsrechtlichen Stellung der Auxiliare einerseits, der Flottensoldaten und equites singulares andererseits zu suchen.

Die Zunahme der cives in den auxilia zeigt uns die jetzt übliche Formel der Auxiliardiplome ${ }^{2}$ ): civitatem R. qui eorum non haberent dedit, während die Diplome der Flottensoldaten den alten Wortlaut beibehalten (D. 62 aus dem Jahre 152). Einen guten Beweis für diese Veränderung erbringen die oben besprochenen Urkunden. Derselbe wiegt um so höher, als wir es hier mit ägyptischen Truppentheilen zu thun haben, in denen doch später als in denen aller übrigen Provinzen Bürger erscheinen. Wir finden in den vier Urkunden, in denen es sich sicher um Auxiliare handelt (Pap. Cattaoui Col. II : vor dem Jahre 115; Pap.

1) Seit der Uebertragung der Vortheile und Nachtheile der "leges" auf das matrimonium iniustum und peregrinum im Anfang des 3. Jahrhunderts wird nur eine den Anforderungen derselben entsprechende Ehe sanctionirt. Die uns erhaltenen Diplome der Flottensoldaten und equites singulares (s. Conc. 119) aus dem 3. Jahrhundert weisen einerseits auf die den Soldaten durch Severus ertheilte Erlaubniss, mit den „Frauen“ im Lager zusammenzuwohnen (Conc. 95 ff.; Kübler 1. 1. S. 362 ff. hat mich durch seine Gegenausführungen nicht überzeugt), andererseits gewähren sie nur einem qualificirten matrimonium peregrinum nachträgliche Legitimation (cum mulieribus, quas secum concessa consuetudine vixisse probaverint). „Peregriner" Concubinat kommt als Ersatzinstitut erst seit dieser Zeit in Betracht, doch nur für Flottensoldaten (C.I.L. VI, 3104; 3158; D. 91) und equites singulares. Die auxiliarii sind jetzt cives, die milites castellani (s. S. 55) haben unbeschränktes Eherecht. - 2) Auch schon vor dem Jahre 142 finden wir in den Diplomen der Auxiliare cives $R$. genannt, die bei der Entlassung also nur conubium erhalten (D. 14: a. d. J. 82; D. 48: a. d. J. 134; s. Mommsen, Hermes 19, 27 A. 1), obwohl die allgemeine Formel lautet: ipsis liberis posterisque eorum civitatem dedit et conubium. Erst das Ueberhandnehmen der cives hat die Aenderung der Formel herbeigeführt. Dje Meisten werden das Bürgerrecht erst bei ihrem Eintritt oder gar während der Dienstzeit erhalten haben. An dem Grundsatz, dass kein Bürger weder gezwungen noch freiwillig in Peregrinencorps eintreten kōnne (Mommsen, Hermes 16, 461 u. 470; 19, 40 u. 60 A. 2) hat man nicht mehr so streng festgehalten, da eben der Charakter des Peregrinencorps sich verlor (s. Eph. ep. VII p. 456 sqq.: s. S. 73). 
Die ägypt. Urkunden und das Eherecht der röm. Soldaten.

Catt. Col. III: J. 115; U.B.M. 114 v. 14 sqq.: J. 134 ; Pap. Catt. C. IV: J. 142): 1 civis R., 2 cives Alexandrini (die eherechtlich den römischen Bürgern gleichstehen) und nur 1 peregrinus.

In den beiden anderen Urkunden (aus deren Text nicht hervorgeht, ob es sich um Legionare oder Auxiliare handelt, obwohl Letzteres wahrscheinlicher ist) sind beide Soldaten cives R. (U.B.M. 114 v. 5 sqq.: Jahr 117; Pap. Catt. Col. V: Jahr 136).

In vollkommenem Gegensatz zu dieser Zusammensetzung der auxilia steht dagegen im 2. Jahrhundert und noch später die der Flotte und der equites singulares. Wenn auch erstere seit Claudius, letztere seit Hadrian zu anerkannten Truppencorps erhoben wurden (Mommsen, Hermes 19, 29), Bürger wurden selbst nach Caracalla kaum zugelassen (nur in den Chargen). Die Rekruten erbalten bei ihrem Eintritt in diese Truppentheile seit Hadrian meistens das ius Latii (Marquardt, St.-V. II, 510 f.; 488 ff.).

Das Motiv der veränderten Stellung der Militärgesetze zum matrimonium iuris peregrini der Auxiliare ist also in dem Bestreben $\mathrm{zu}$ erblicken, die Minorität, die noch peregrinae condicionis war, während der Dienstzeit mit gleichem Mass zu messen, wie die eberechtlich schon immer benachtheiligten cives R., die jetzt die Majorität der Auxiliartruppen bilden.

Um die Mitte des 2. Jahrhunderts sind kaum mehr Peregrine in den auxilia vorhanden. Das zeigt uns die Eph. ep. VII p. $456 \mathrm{sqq}$. von Mommsen veröffentlichte Zugangsliste der Soldaten der cohors I. Aug. pr. Lusitanorum aus dem Jahre 156 (s. auch U.B.M. 462 aus der Zeit des Antoninus Pius; U.B.M.447 aus den Jahren 173/175): Unter 16 Soldaten der Cohorte, deren Namen uns einigermassen erhalten sind, befinden sich nur 4, welche auch jetzt noch ihren peregrinen Stand beibehalten haben $(\mathrm{I}, 38 ; \mathrm{I}, 43 ; \mathrm{II}, 5 ; \mathrm{II}, 35)$ und auch nach ihrem Diensteintritt (bis auf einen sind alle tirones voluntarii) Aegypter geblieben sind.

Die übrigen sind cives R., und zwar nicht etwa durchweg peregrine Rekruten, die erst bei ihrem Eintritt in das Heer die civitas erbalten haben. Vielmehr werden auch schon im Besitz des Bürgerrechts Befindliche in die Cohorte eingestellt, so der reiectus ab ala $A$. Flavius Vespasianus (I, 29), der zwar decurio ist; wohl auch der translatus ex coh. I. Fl. 
Cil. Maevius Marcellus $(\mathrm{II}, 24)^{1}$ ), vor Allem die accepti ex leg. II. Tr. Fort. (II, 13-21). Die Auxiliarcohorte erscheint danach zwar noch immer minderwerthiger als die ala ${ }^{2}$ ), sie wird aber nicht mehr als ein grundsätzlich von der bürgerlichen Legion verschiedener Truppentheil betrachtet. Es findet ein Austausch $z$ wischen beiden statt: Die Auxiliarveteranen bilden einen Theil des Rekrutirungsmateriales für die ägyptischen Legionen (U.B.M. 113; 265); andererseits werden Legionare ohne Avancement in die Cohorten versetzt ${ }^{3}$ ).

Die Ausgleichung zwischen den früheren Peregrinencorps der auxilia und den Bürgertruppen ist fast vollzogen. An Stelle der Scheidung in legiones und auxilia tritt in steter Weiterentwickelung eine solche in reguläre Bürgertruppen (legiones, auxilia) und Milizen (Grenzer, milites castellani: s. S. 55), welche, unter Severus Alexander ausgebildet, am Ende des 3. Jahrhunderts vollendet ist (comitatenses und limitanei).

Und dem entsprechen die eherechtlichen Verhältnisse. Auf der einen Seite Eheverbot für legionarii und auxiliarii, deren Diplome seit dem Jahre 178 aufhören, keine eherechtlichen Privilegien bei der Entlassung. Auf der anderen Seite Eherecht schon während der Dienstzeit (für die filii militum castellanorum: D. 90 unter Severus Alexander).

Das führt schliesslich mit dem Niedergange des regulären Heeres zum Eherecht aller Soldaten des römischen Heeres.

1) $\mathrm{Ob}$ der einzige tiro lectus, der genannt wird (miles factus ex pagano: I, 20-24) und der das nomen des praef. Aeg. trägt, unter dem er ausgemustert ist (probatus, ह̇ $\pi \bullet x \rho \bullet \vartheta \varepsilon i s$ ), nicht erst bei seinem Eintritt die civitas erhalten hat, will jch dahingestellt sein lassen; s. auch U.B.M. 447, wo ein im Jahre 154/155 von demselben praef. Aeg. M. Sempronius Liberalis ausgemusterter eques alae I. Mauretanorum Sempronius Herminus heisst. - ${ }^{2}$ ) Die Soldaten der ala werden in Bezug auf Behandlung und Strafmaass den Legionaren gleichgestellt, während die cohortales auch jetzt noch tiefer stehen (cf. Mommsen Eph. ep. 1. l. p. 465). Es ist dies wohl eine Nachwirkung der früheren Rekrutirung der ägyptischen Alen aus Griechen (Alexandrinern), der Cohorten aus Aegyptern, welche strenger bestraft werden als jene (Mommsen, Röm. Gesch. V, 561

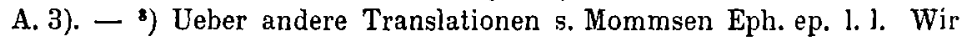
finden zwar auch Translationen aus der cohors II. Ulpia Afrorum in die classis Alexandrina (U.B.M. 142: J. 159). 